\title{
DECOMPOSITIONS INTO CODIMENSION-TWO MANIFOLDS
}

BY

\author{
R. J. DAVERMAN AND J. J. WALSH ${ }^{1}$
}

\begin{abstract}
Let $M$ denote an orientable $(n+2)$-manifold and let $G$ denote an upper semicontinuous decomposition of $M$ into continua having the shape of closed, orientable $n$-manifolds. The main result establishes that the decomposition space $M / G$ is a 2 -manifold.
\end{abstract}

1. Introduction. Let $M$ denote an orientable $(n+2)$-manifold and let $G$ denote an upper semicontinuous decomposition of $M$ into continua having the shape of closed, orientable $n$-manifolds. The main result here establishes that the decomposition space $M / G$ is a 2 -manifold.

This theorem lifts what is known about decompositions into codimension-two submanifolds to nearly the same level as what is known about decompositions into codimension-one submanifolds. Liem [L] proved that if $G$ is a decomposition of an $(n+1)$-manifold $N$ into compacta having the shape of $S^{n}$, then $N / G$ is a 1 -manifold, and for $n>4$ the decomposition map $p: N \rightarrow N / G$ can be approximated by a locally trivial bundle map. Daverman [D] showed that if $G$ is a decomposition of $N$ into continua having the shape of arbitrary closed $n$-manifolds, then $N / G$ is a 1-manifold (possibly with boundary if various orientability conditions are not all met).

In an earlier paper [DW1] we derived the conclusion that $M / G$ is a 2-manifold in case $M$ is an $(n+2)$-manifold and each $g$ in $G$ has the shape of $S^{n}$. Then the natural map $p: M \rightarrow M / G$ must be particularly nice, being an approximate fibration when $n>1$ (the same is "almost" true when $n=1$ ). The present work evolved from techniques similar to those of [DW1], which were inspired for the most part by methods of Coram and Duvall [CD1, CD2]. However, the strong conclusion that $p$ : $M \rightarrow M / G$ is an approximate fibration cannot be attained in the more general situation at hand.

In the fourth section we determine the structure of a certain sheaf $\mathscr{H}^{n}$ on $M / G$ induced by $p$. The properties of this sheaf can be used to recover the conclusions in [DW1] just mentioned, in the special situation where the elements of $G$ have the shape of a $n$-sphere.

Received by the editors April 5, 1984 and, in revised form, July 31, 1984.

1980 Mathematics Subject Classification. Primary 57N15, 57N05; Secondary 55P55, 54B15.

Key words and phrases. Codimension-two submanifold, winding function, upper semicontinuous decomposition, 2-manifold.

${ }^{1}$ Research of the first author was supported in part by NSF Grant MCS 81-20741; research of the second author was supported in part by NSF Grant MCS 82-00913 and Alfred P. Sloan Foundation Grant. 
The manifold features present in such decomposition spaces $M / G$ do not persist through successively larger codimensions. If $G$ is a decomposition of a manifold $M$ into codimension-three submanifolds, $M / G$ need not be a manifold nor need it even be a generalized manifold. It seems that the fundamental question to address is whether $M / G$, if finite dimensional, is an ANR.

It should be pointed out that, in our terminology, a manifold is separable, metric and boundaryless. We will speak of a manifold with boundary as the need to permit a boundary occurs.

The symbol $\approx$ will be used to mean "is homeomorphic to" and the symbol $\cong$ will be used to mean "is isomorphic to." Unless expressly stated otherwise, homology and cohomology groups are determined with integral $(\mathbf{Z})$ coefficients.

2. The winding function and its continuity set. Throughout the remainder of the paper we shall employ the following notation: $M$ for an orientable $(n+2)$-manifold, $n \geqslant 1, G$ for a usc (the standard abbreviation of "upper semicontinuous") decomposition of $M$ into compact connected elements each having the shape of a closed orientable $n$-manifold, $B$ for the decomposition space $M / G$, and $p$ for the decomposition map $M \rightarrow B$.

We begin with a variation on the Coram-Duvall definition of local winding number function. Temporarily fix $b_{0} \in B$. Since $g_{0}=p^{-1} b_{0}$ is movable, there exist connected neighborhoods $U$ and $U_{0}$ of $b_{0}$ in $B$ such that $b_{0} \in U_{0} \subset U$ and (the inclusion induced)

$$
\phi_{0}: \check{H}_{n}\left(p^{-1} b_{0}\right) \rightarrow H_{n}\left(p^{-1} U\right)
$$

is an isomorphism onto the image of

$$
\phi: H_{n}\left(p^{-1} U_{0}\right) \rightarrow H_{n}\left(p^{-1} U\right)
$$

For any $b \in U_{0}$, the image of

$$
\phi_{h}: \check{H}_{n}\left(p^{-1} b\right) \rightarrow H_{n}\left(p^{-1} U\right)
$$

is contained in $\operatorname{Im} \phi$. Hence,

$$
\phi_{0}^{-1} \phi_{b}: \check{H}_{n}\left(p^{-1} b\right) \rightarrow \check{H}_{n}\left(p^{-1} b_{0}\right)
$$

is a well-defined homomorphism between copies of $\mathbf{Z}$, meaning that it amounts to multiplication by some integer $q_{h} \geqslant 0$. Define the winding function $\alpha: U_{0} \rightarrow \mathbf{Z}$ by $\alpha(b)=q_{b}$.

Let $K$ denote the set of all points $b_{0}$ in $B$ such that every neighborhood $W$ of $b_{0}$ (with $W \subset U_{0}$ ) contains some point $w$ for which $\alpha(w)=0$. As in previous analyses in [CD1, CD2, DW1], the set $K$ is a troublesome spot. Clearly, $K$ is closed in $B$.

\section{LEMMA 2.1. $K$ is nowhere dense in $B$.}

Proof. See [CD2, Lemma 3.1].

Define $B_{+}$as $B-K$. In the rest of this section we outline an argument showing that the complement in $B_{+}$of a certain countable closed subset is a 2-manifold. Although operating under less limiting hypotheses, we are able to extract enough from arguments in [CD1 and DW1, §3] to satisfy our needs. 
We shall continue to work locally throughout most of this section. Assume that $b_{0} \in B$ mentioned near the beginning lies in $B_{+}$and that the neighborhood $U_{0}$ mentioned there satisfies $U_{0} \subset B_{+}$and

$$
\operatorname{Im}\left\{\check{H}_{1}\left(p^{-1} b_{0}\right) \rightarrow H_{1}\left(p^{-1} U\right)\right\}=\operatorname{Im}\left\{H_{1}\left(p^{-1} U_{0}\right) \rightarrow H_{1}\left(p^{-1} U\right)\right\}
$$

as well. The three facts stated below are direct analogues of Lemmas 1-3 in [CD1].

(1) If $b \in U_{0}$, then there is a neighborhood $V$ of $b$ in $U_{0}$ such that to each $b^{\prime} \in V$ there corresponds a positive integer $k$ such that $\alpha\left(b^{\prime}\right)=k \alpha(b)$.

(2) $\alpha$ is lower semicontinuous.

(3) The continuity set $C=\left\{b \in U_{0}: \alpha\right.$ is continuous at $\left.b\right\}$ is open and dense in $U_{0}$; its complement $D=U_{0}-C$ can be written as $D_{1} \cup D_{2}$ where $D_{2}$ is countable, $D_{1}$ is closed, and each neighborhood of $d \in D_{1}$ contains uncountably many points of $D_{1}$.

(Here $D_{2}=\{d \in D$ : some neighborhood of $d$ in $D$ is countable $\}$ and $D_{1}=D-$ $D_{2}$.)

LeMma 2.2. Let $A$ be an arc in $C$. Then, using either $\mathbf{Z}$ or $\mathbf{Z} / 2 \mathbf{Z}$-coefficients,

(i) $\check{H}^{n+1}\left(p^{-1} A\right) \cong 0$;

(ii) for $x \in A, i^{*}: \check{H}^{n}\left(p^{-1} A\right) \rightarrow \check{H}^{n}\left(p^{-1} x\right)$ is surjective; and

(iii) for $x, y \in A, \operatorname{ker}\left\{\check{H}^{n}\left(p^{-1} A\right) \rightarrow \check{H}^{n}\left(p^{-1} x\right)\right\}=\operatorname{ker}\left\{\check{H}^{n}\left(p^{-1} A\right) \rightarrow \check{H}^{n}\left(p^{-1} y\right)\right\}$.

Proof. We assume the coefficient group is $\mathbf{Z}$, the argument being the same for other coefficient groups. For each $x \in A$, there is a closed subarc $A_{x}$, a neighborhood of $x$ in $A$, for which there is a shape strong deformation retraction of $p^{-1} A_{x}$ to $p^{-1} x$, the deformation occurring in a neighborhood of $p^{-1} x$. Consequently, the diagram

$$
\check{H}^{n}\left(p^{-1} y\right) \quad i^{*} \swarrow \quad \stackrel{\check{H}^{n}\left(p^{-1} A_{x}\right)}{ } \quad \searrow i^{*}
$$

commutes for each $y \in A_{x}$, where $\psi$ is induced by the retraction and is an isomorphism as we are working in the continuity set. It follows easily that the $\operatorname{arc} A_{x}$ satisfies conclusions (ii) and (iii).

If a closed subarc $A^{\prime}$ of $A$ satisfies conclusion (ii), then obviously so does each closed subarc $A^{\prime \prime}$; moreover, inclusion induces a surjection

$$
\check{H}^{n}\left(p^{-1} A^{\prime}\right) \rightarrow \check{H}^{n}\left(p^{-1} A^{\prime \prime}\right) .
$$

To see why this is so, express $A^{\prime}=E_{1} \cup A^{\prime \prime} \cup E_{2}$ as the union of closed subarcs (one of $E_{1}$ or $E_{2}$ being empty, should $A^{\prime \prime}$ contain an endpoint of $A^{\prime}$ ), where $E_{1} \cap A^{\prime \prime}$ and $E_{2} \cap A^{\prime \prime}$ are endpoints of $A^{\prime \prime}$. Given an element $z \in \check{H}^{n}\left(p^{-1} A^{\prime \prime}\right)$, use conclusion (ii) to find $z_{j} \in \check{H}^{n}\left(p^{-1} E_{j}\right)$ for $j \in\{1,2\}$ such that

$$
\operatorname{Im}\left\{z_{j} \rightarrow \check{H}^{n}\left(p^{-1}\left(A^{\prime \prime} \cap E_{j}\right)\right)\right\}=\operatorname{Im}\left\{z \rightarrow \check{H}^{n}\left(p^{-1}\left(A^{\prime \prime} \cap E_{j}\right)\right)\right\} .
$$

From the Mayer-Vietoris sequence of $\left(p^{-1}\left(A^{\prime \prime} \cup E_{1}\right), p^{-1} A^{\prime \prime}, p^{-1} E_{1}\right)$,

$$
\check{H}^{n}\left(p^{-1}\left(A^{\prime \prime} \cup E_{2}\right)\right) \rightarrow \check{H}^{n}\left(p^{-1} A^{\prime \prime}\right) \oplus \check{H}^{n}\left(p^{-1} E_{1}\right) \stackrel{\Delta}{\rightarrow} \check{H}^{n}\left(p^{-1}\left(A^{\prime \prime} \cap E_{1}\right)\right),
$$


we see that $\Delta\left(z \oplus z_{1}\right)=0$, which gives $z^{\prime \prime} \in \check{H}^{n}\left(p^{-1}\left(A^{\prime \prime} \cup E_{1}\right)\right)$, whose restrictions ( = inclusion-induced images) in $\check{H}^{n}\left(p^{-1} A^{\prime \prime}\right)$ and $\check{H}^{n}\left(p_{1}^{-1}\right)$ are $z$ and $z_{1}$, respectively. Similarly, starting from part of the Mayer-Vietoris sequence for

$$
\left(p^{-1}\left(E_{1} \cup A^{\prime \prime} \cup E_{2}\right), p^{-1}\left(E_{1} \cup A^{\prime \prime}\right), p^{-1} E_{2}\right),
$$

we obtain $z^{\prime} \in H^{\prime \prime}\left(p^{-1} A^{\prime}\right)$ whose restriction to $H^{n}\left(p^{-1} A^{\prime \prime}\right)$ is $z$. It now follows easily that if, in addition, $A$ satisfies conclusion (iii), then so does each closed subarc.

Let $A_{1}$ and $A_{2}$ denote closed subarcs of $A$ such that $A_{1} \cap A_{2} \neq \varnothing$. By the preceding argument, if $A_{1}$ and $A_{2}$ satisfy conclusion (ii), so does their union $A_{1} \cup A_{2}$. If both $A_{1}$ and $A_{2}$ satisfy conclusion (iii), we can verify that the same is true of $A$ by supposing $y \in A_{1} \cap A_{2}$ and $x \in A_{1}$, say, and considering the commutative diagram:

$$
\begin{aligned}
& \check{H}^{n}\left(p^{-1} A\right)
\end{aligned}
$$

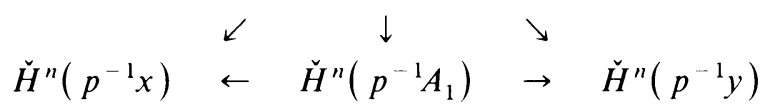

Let $A^{\prime}$ be a maximal subarc of $A$ such that conclusions (ii) and (iii) are valid for each proper closed subarc of $A^{\prime}$. Each endpoint of $A^{\prime}$ is contained in $A^{\prime}$, else the arc $A_{x}$, where $x$ is an endpoint of $A^{\prime}$, together with the conclusions of the previous paragraph would lead to a contradiction of the maximality of $A^{\prime}$. For the same reason, $A^{\prime}=A$.

Finally, conclusion (i) is a consequence of conclusion (ii) since repeated applications of the Mayer-Vietoris sequence reveal

$$
\check{H}^{n+1}\left(p^{-1} A\right) \cong \check{H}^{n+1}\left(p^{-1} A_{1}\right) \oplus \cdots \oplus \check{H}^{n+1}\left(p^{-1} A_{k}\right)
$$

for any "partition" $A_{1}, \ldots, A_{k}$ of $A$ into subarcs. The nontriviality of $\check{H}^{n+1}\left(p^{-1} A\right)$ would ultimately detect the nontriviality of $\check{H}^{n+1}\left(p^{-1} x\right)$ for some $x \in A$, but this is not possible.

LemMA 2.3. Neither a point nor an arc in $C$ separates $C$, but every simple closed curve there does separate $C$.

Proof. Let $A$ denote an arc in $C$ and $V$ the component of $C$ containing $A$. From the exact sequence of the pair $\left(p^{-1} V, p^{-1} V-p^{-1} A\right)$, we obtain

$$
H_{1}\left(p^{-1} V, p^{-1} V-p^{-1} A\right) \rightarrow \tilde{H}_{0}\left(p^{-1} V-p^{-1} A\right) \rightarrow \tilde{H}_{0}\left(p^{-1} A\right) \cong 0
$$

and, by duality [S, p. 296] and Lemma 2.2,

$$
H_{1}\left(p^{-1} V, p^{-1} V-p^{-1} A\right) \cong \check{H}^{n+1}\left(p^{-1} A\right) \cong 0 \text {. }
$$

Thus $p^{-1} V-p^{-1} A$ and $V-A$ are connected.

Similarly, no point separates $C$.

Now, let $J$ denote a simple closed curve in some component $V$ of $C$. Express $J$ as the union of two $\operatorname{arcs} A_{1}$ and $A_{2}$ whose intersection is the boundary $\left\{a_{1}, a_{2}\right\}$ of each. From the Mayer-Vietoris sequence for $p^{-1} J=p^{-1} A_{1} \cup p^{-1} A_{2}$, we analyse with $\mathbf{Z} / 2 \mathbf{Z}$-coefficients

$$
\begin{gathered}
\cdots \rightarrow \check{H}^{n}\left(p^{-1} A_{1}\right) \oplus \check{H}^{n}\left(p^{-1} A_{2}\right) \stackrel{\beta}{\rightarrow} \check{H}^{n}\left(p^{-1}\left\{a_{1}, a_{2}\right\}\right) \rightarrow \check{H}^{n+1}\left(p^{-1} J\right) \rightarrow 0 \\
\cong \downarrow \phi \\
\check{H}^{n}\left(p^{-1} a_{1}\right) \oplus \check{H}^{n}\left(p^{-1} a_{2}\right) \cong \mathbf{Z} / 2 \mathbf{Z} \oplus \mathbf{Z} / 2 \mathbf{Z}
\end{gathered}
$$


(where the zero at the right comes from Lemma 2.2). Write $\phi \circ \beta=\delta_{1} \oplus \delta_{2}$, where, for $i=1,2, \delta_{i}: \check{H}^{n}\left(p^{-1} A_{1}\right) \oplus \check{H}^{n}\left(p^{-1} A_{2}\right) \rightarrow \check{H}^{n}\left(p^{-1} a_{i}\right)$. The conclusion in part (iii) of Lemma 2.2 reveals that $\delta_{1}(z, 0)=0$ if and only if $\delta_{2}(z, 0)=0$ and, likewise, that $\delta_{1}(0, z)=0$ if and only if $\delta_{2}(0, z)=0$. It follows that the image of $\phi \beta$ is contained in the diagonal subgroup $\{(0,0),(1,1)\}$ and, in light of part (ii) of Lemma 2.2, is equal to this subgroup. Consequently,

$$
\check{H}^{n+1}\left(p^{-1} J ; \mathbf{Z} / 2 \mathbf{Z}\right) \cong \mathbf{Z} / 2 \mathbf{Z} \text {. }
$$

Continuing throughout the remainder of the proof to use $\mathbf{Z} / 2 \mathbf{Z}$-coefficients, we establish that $p^{-1} J$ separates $p^{-1} V$ by showing that the homomorphism $\gamma$ in the sequence below is trivial:

$$
\begin{aligned}
& H_{1}\left(p^{-1} V\right) \stackrel{\gamma}{\rightarrow} \quad H_{1}\left(p^{-1} V, p^{-1} V-p^{-1} J\right) \rightarrow \tilde{H}_{0}\left(p^{-1} V-p^{-1} J\right) \quad \rightarrow \quad 0 \\
& \downarrow \cong \\
& \check{H}^{n+1}\left(p^{-1} J\right) \cong \mathbf{Z} / 2 \mathbf{Z}
\end{aligned}
$$

A simple chase through the diagram

$$
\begin{array}{ccccc}
H_{1}\left(p^{-1} V-p^{-1} J\right) & \rightarrow & H_{1}\left(p^{-1} V\right) & \stackrel{\gamma}{\rightarrow} & H_{1}\left(p^{-1} V, p^{-1} V-p^{-1} J\right) \\
\downarrow & & \downarrow & & \\
H_{1}\left(p^{-1} U_{0}-p^{-1} J\right) & \rightarrow & H_{1}\left(p^{-1} U_{0}\right) & \rightarrow & H_{1}\left(p^{-1} U_{0}, p^{-1} U_{0}-p^{-1} J\right) \\
\downarrow & & j \downarrow & & \\
H_{1}\left(p^{-1} U-p^{-1} J\right) & \stackrel{\eta}{\rightarrow} & H_{1}\left(p^{-1} U\right) & \stackrel{\gamma^{\prime}}{\rightarrow} & H_{1}\left(p^{-1} U, p^{-1} U-p^{-1} J\right)
\end{array}
$$

establishes the triviality of $\gamma$ provided $\operatorname{Im} \eta \supset \operatorname{Im} j$. One can detect the last containment by choosing $c_{0} \in U_{0}-J$ so close to $b_{0}$ that $c_{0}$ lies in a neighborhood $W$ of $b_{0}$ for which there is a shape deformation retraction in $p^{-1} U_{0}$, say $\underline{r}: p^{-1} W \rightarrow p^{-1} b_{0}$. Since the restriction of $\underline{r}$ induces an isomorphism $\check{H}_{n}\left(p^{-1} c_{0}\right) \rightarrow \check{H}_{n}\left(p^{-1} b_{0}\right)$ with $\mathbf{Z}$ and, hence, with $\mathbf{Z} / 2 \mathbf{Z}$-coefficients (as we are working in the continuity set), $\underline{r}$ induces a surjection $\check{H}_{1}\left(p^{-1} c_{0}\right) \rightarrow \check{H}_{1}\left(p^{-1} b_{0}\right)$. It follows that

$$
\operatorname{Im}\left\{\check{H}_{1}\left(p^{-1} c_{0}\right) \rightarrow H_{1}\left(p^{-1} U\right)\right\}=\operatorname{Im}\left\{\check{H}_{1}\left(p^{-1} b_{0}\right) \rightarrow H_{1}\left(p^{-1} U\right)\right\} .
$$

We stipulated earlier (for $\mathbf{Z}$ and, hence, $\mathbf{Z} / 2 \mathbf{Z}$-coefficients) that

$$
\operatorname{Im}\left\{\check{H}_{1}\left(p^{-1} b_{0}\right) \rightarrow H_{1}\left(p^{-1} U\right)\right\}=\operatorname{Im}\left\{H_{1}\left(p^{-1} U_{0}\right) \rightarrow H_{1}\left(p^{-1} U\right)\right\},
$$

and, as

$$
\operatorname{Im} \eta \supset \operatorname{Im}\left\{\check{H}_{1}\left(p^{-1} c_{0}\right) \rightarrow H_{1}\left(p^{-1} U\right)\right\},
$$

we conclude that $\operatorname{Im} \eta \supset \operatorname{Im} j$.

ADDENDUM. Having established that $p^{-1} J$ separates, we can conclude that $\check{H}^{n+1}\left(p^{-1} J ; \mathbf{Z}\right) \cong \mathbf{Z}$. The only point in the preceding argument that made essential use of $\mathbf{Z} / 2 \mathbf{Z}$-coefficients arose in the conclusion that the image of $\beta$ equalled a diagonal subgroup. For $\mathbf{Z}$-coefficients, we can draw the analogous conclusion provided we know $\check{H}^{n+1}\left(p^{-1} J ; \mathbf{Z}\right) \neq 0$, which holds because $p^{-1} J$ separates $V$ (and since the homomorphism $\gamma$ analysed in the proof is trivial for Z-coefficients as well). 
THEOREM 2.4. The space $C$ is a 2-manifold.

Proof. This is a consequence of Lemma 2.3 and [Wi, p. 95].

LEMMa 2.5. The set $D$ is countable.

Proof. By fact (3), $D=D_{1} \cup D_{2}$ where $D_{1}$ is closed in $D$ and dense-in-itself, and where $D_{2}$ is countable. We shall prove that $D_{1}=\varnothing$.

Suppose $D_{1} \neq \varnothing$. Since $\alpha \mid D_{1}$ is lower semicontinuous, the set where $\alpha \mid D_{1}$ is continuous forms a dense open subset of $D_{1}$. Thus, we can find $b \in D_{1}$ and some neighborhood $W$ of $b$ in $U_{0}$ such that $\alpha \mid D_{1} \cap W$ is constant. Accordingly, as in fact (1), $W$ can be restricted so that for all $w \in W$ there exists an integer $k(w)>0$ such that $\alpha(w)=k(w) \cdot \alpha(b)$. Since $b$ is a discontinuity of $\alpha$, there is some $w \in W-D_{1}$ for which $k(w)>1$. This yields $k\left(w^{\prime}\right) \geqslant k(w)>1$ for all $w^{\prime}$ in some neighborhood of $w$, so we can assume $w \in W-D$.

Using the countability of $D_{2}$, we build an arc $A$ in $W$ with endpoints $c, d$ in $D_{1}$ and with $A-\{c, d\} \subset W-D$ such that, for $x \in A-\{c, d\}$,

$$
\alpha(x)=k \alpha(c)=k \alpha(d) \quad(\text { some } k>1) .
$$

It suffices to compute that $\check{H}^{n+1}\left(p^{-1} A\right) \cong \mathbf{Z} / k \mathbf{Z}$, for one can verify that this is impossible by inspecting the sequence

$$
\begin{gathered}
H_{1}\left(p^{-1} W\right) \stackrel{\gamma}{\rightarrow} H_{1}\left(p^{-1} W, p^{-1} W-p^{-1} A\right) \rightarrow \tilde{H}_{0}\left(p^{-1} W-p^{-1} A\right) \rightarrow 0 \\
\downarrow \cong \\
\check{H}^{n+1}\left(p^{-1} A\right) \cong \mathbf{Z} / k \mathbf{Z}
\end{gathered}
$$

while recalling the argument in Lemma 2.3 that $\gamma$ is the trivial homomorphism.

Express $A$ as the union of two subarcs $A_{1}, A_{2}$ having a common endpoint $e$. It suffices to determine that, for $i=1,2, \check{H}^{n+1}\left(p^{-1} A_{i}\right) \cong 0$ and that

$$
\operatorname{Im}\left\{\check{H}^{n}\left(p^{-1} A_{i}\right) \rightarrow \check{H}^{n}\left(p^{-1} e\right)\right\}=k \mathbf{Z} .
$$

Then the tail of a Mayer-Vietoris sequence

$$
\check{H}^{n}\left(p^{-1} A_{1}\right) \oplus \check{H}^{n}\left(p^{-1} A_{2}\right) \rightarrow \check{H}^{n}\left(p^{-1} e\right) \rightarrow \check{H}^{n+1}\left(p^{-1} A\right) \rightarrow 0
$$

detects that $\check{H}^{n+1}\left(p^{-1} A\right) \cong \mathbf{Z} / k \mathbf{Z}$.

Sublemma 2.6. Let $W$ and $D$ be as above and let $A$ be an arc with one endpoint $c \in D$, with $A \backslash\{c\} \subset W$, and with $\alpha(x)=k \alpha(c)$ for $x \in A-\{c\}$ (some $k>1)$. Then

(i) $\check{H}^{n+1}\left(p^{-1} A\right)=0$, and

(ii) $\operatorname{Im}\left\{\check{H}^{n}\left(p^{-1} A\right) \rightarrow \check{H}^{n}\left(p^{-1} e\right)\right\}=k \mathbf{Z}$, where $e$ is the endpoint of $A-\{c\}$.

Proof. Conclusion (i) follows from conclusion (ii) of Lemma 2.2, just as conclusion (i) of Lemma 2.2 followed. (The key observation is that, expressing $A=A_{1} \cup A_{2}$ as the union of subarcs having just an endpoint in common,

$$
\check{H}^{n}\left(p^{-1} A_{1}\right) \oplus \check{H}^{n}\left(p^{-1} A_{2}\right) \rightarrow \check{H}^{n}\left(p^{-1}\left(A_{1} \cap A_{2}\right)\right)
$$

is surjective, since one of $A_{1}$ and $A_{2}$ is contained in the continuity set.) 
Take an arbitrary neighborhood $N$ of $p^{-1} A$. Let $A_{1}$ be a proper subarc of $A$ containing $c$ for which there exists a shape strong deformation of $p^{-1} A_{1}$ to $p^{-1} c$, the deformation taking place in $N$. Let $d$ denote the other endpoint of $A_{1}$ and $A^{\prime}=$ $\mathrm{Cl}\left(A-A_{1}\right)$. It follows that

$$
\operatorname{Im}\left\{\check{H}^{n}\left(p^{-1} A_{1}\right) \rightarrow \check{H}^{n}\left(p^{-1} d\right)\right\}=k \mathbf{Z}
$$

because $\alpha(d)=k \alpha(c)$. From the cohomology ladder

$$
\begin{array}{ccccccc}
\check{H}^{n}\left(p^{-1} A\right) & \rightarrow & \check{H}^{n}\left(p^{-1} A^{\prime}\right) & \rightarrow & \check{H}^{n+1}\left(p^{-1} A, p^{-1} A^{\prime}\right) & \rightarrow & 0 \\
\downarrow & & \downarrow & & & \downarrow \cong & \\
\check{H}^{n}\left(p^{-1} A_{1}\right) & \rightarrow & \check{H}^{n}\left(p^{-1} d\right) & \rightarrow & \check{H}^{n+1}\left(p^{-1} A_{1}, p^{-1} d\right) & \rightarrow & 0 \\
& & \downarrow \cong & & & \downarrow \cong & \\
& & \mathbf{Z} & & & \mathbf{Z} / k \mathbf{Z} &
\end{array}
$$

we see that $\operatorname{Im}\left\{\check{H}^{n}\left(p^{-1} A\right) \rightarrow \check{H}^{n}\left(p^{-1} d\right)\right\}=k \mathbf{Z}$. The desired conclusion that

$$
\operatorname{Im}\left\{\check{H}^{n}\left(p^{-1} A\right) \rightarrow \check{H}^{n}\left(p^{-1} e\right)\right\}=k \mathbf{Z}
$$

follows from the following diagram and the conclusion of Lemma 2.2 that the surjections $i_{e}^{*}$ and $i_{d}^{*}$ have equal kernels:

$$
\begin{array}{ccccc} 
& \multicolumn{5}{c}{\check{H}^{n}\left(p^{-1} A\right)} & & \\
& \swarrow & \downarrow & \searrow & \\
\check{H}^{n}\left(p^{-1} e\right) & \stackrel{i_{*}^{*}}{\leftarrow} & \check{H}^{n}\left(p^{-1} A^{\prime}\right) & \stackrel{i_{d}^{*}}{\rightarrow} & \check{H}^{n}\left(p^{-1} d\right)
\end{array}
$$

This completes the proof that $D$ is countable.

Now it is appropriate to let the point $b_{0}$, identified shortly after the statement of Lemma 2.1, take on different values in $B_{+}=B-K$. For each $b \in B_{+}$we have a neighborhood $U_{b} \subset B_{+}$, a closed and countable subset $D_{b}$ of $U_{b}$, a dense open subset $C_{b}=U_{b}-D_{b}$ of $U_{b}$, and a winding function $\alpha_{b}: U_{b} \rightarrow \mathbf{Z}$ with the properties described at the outset of this section. In case $c \in U_{b(1)} \cap U_{b(2)}$, then $c \in C_{b(1)}$ iff $c \in C_{b(2)}$, since continuity of $\alpha_{b(i)}$ at $c$ depends only on the behavior of point inverses near $p^{-1} c$. Select a countable subcollection $\left\{U_{b(i)}\right\}$ of $\left\{U_{b}\right\}$ covering $B_{+}$and define $C_{+}$as $\bigcup_{i} C_{b(i)}$. From Theorem 2.4 and Lemma 2.5 we obtain

Theorem 2.7. The set $C_{+}$is open and dense in $B$ and $D_{+}=B_{+}-C_{+}$is a countable, relatively closed subset of $B_{+}$. Furthermore, $C_{+}$is a 2-manifold.

3. The structure of the decomposition space. At this point we launch an investigation of the subset $K=B-B_{+}$, with an eye toward ultimately concluding it is locally finite. The crucial step is detecting that $K$ is totally disconnected, for we have

LEMMA 3.1. If $K$ is totally disconnected, then $B$ is a 2-manifold.

Sketch of The Proof. The argument in Lemma 2.3 shows each $y_{0} \in K \cup D_{+}$to have a connected neighborhood $U_{0}$ in $B$ such that every simple closed curve in $U_{0} \cap C_{+}$separates $U_{0} \cap C_{+}$. Thus, as argued in [DW1, Lemma 4.1], $U_{0} \cap C_{+}$is homeomorphic to the complement in $S^{2}$ of some closed 0-dimensional subset. The 
points of $\left(K \cup D_{+}\right) \cap U_{0}$ can be considered to be (some of the) ends of $U_{0}$ and the homeomorphism of $U_{0} \cap C_{+}$into $S^{2}$ can be extended, in an end-preserving way, to one from $U_{0}$ onto an open subset of $S^{2}$.

Suppose now that $K_{1} \subset K$ is a closed subset such that $K-K_{1}$ is totally disconnected (equivalently, 0 -dimensional, as $K-K_{1}$ is locally compact). Using [DH, Theorem 2.10], we name a point $b_{0} \in K_{1}$, a neighborhood $U_{0}$ of $b_{0}$ as above, and then another neighborhood $W$ of $b_{0}$ in $U_{0}$ for which there exists a shape retraction $\underline{r}$ : $p^{-1} W \rightarrow p^{-1} b_{0}$ such that the restriction of $\underline{r}$ to $p^{-1} b$ provides a shape equivalence from $p^{-1} b$ to $p^{-1} b_{0}$ for each $b \in K_{1} \cap W$.

Proposition 3.2. There is a nonempty relatively open subset $\mathcal{O}$ of $K_{1} \cap W$ that is totally disconnected (equivalently, in this setting, 0-dimensional).

Before embarking on the proof, which occupies most of the remainder of this section, we derive the important

COROllary 3.3. $K$ is totally disconnected.

Proof. Specify $K_{1}$ by requiring $K-K_{1}$ to be a maximal relatively open subset of $K$ that is 0 -dimensional. The separability of $K$ and the Sum Theorem for 0 -dimensional sets [HW, p. 18] ensure that such a subset exists. Then $K_{1}=\varnothing$, for otherwise a nonempty, 0 -dimensional, relatively open subset $\mathcal{O}$ of $K_{1}$ would exist, and ( $K-$ $\left.K_{1}\right) \cup \mathcal{O}$ would be larger than $K-K_{1}$. It would be obvious that $\left(K-K_{1}\right) \cup \mathcal{O}$ is relatively open in $K$ and the Sum Theorem of [HW] again would reveal that $\left(K-K_{1}\right) \cup \mathcal{O}$ is 0 -dimensional. Thus, $K$ itself is 0 -dimensional.

LEMMA 3.4. There is a connected neighborhood $W_{1} \subset W$ of $b_{0}$ and a component $V$ of $W_{1}-K_{1}$ such that every arc $A$ with $\partial A \subset K_{1}$ and $A-\partial A \subset V \cap C_{+}$separates $B$ and one component of $B-A$ is contained in $U_{0}$.

Proof. Choose $W_{1} \subset W$ so that $H_{n+1}\left(p^{-1} W_{1}\right) \rightarrow H_{n+1}\left(p^{-1} U_{0}\right)$ is trivial. Since $b_{0} \in K$, there exists $w \in W_{1}$ such that $\check{H}_{n}\left(p^{-1} w\right) \rightarrow H_{n}\left(p^{-1} W_{1}\right)$ is trivial. Because $\alpha \mid K_{1} \cap W$ is nonzero, $w \notin K_{1}$. It follows from the 0-dimensionality of [( $\left.K-K_{1}\right) \cup$ $\left.D_{+}\right] \cap W_{1}$ (and from the fact that $\check{H}_{n}\left(p^{-1} w^{\prime}\right) \rightarrow H_{n}\left(p^{-1} W_{1}\right)$ is trivial for all $w^{\prime}$ in some neighborhood of $w$ ) that we can choose $w \in W_{1} \cap C_{+}$.

Let $V$ denote the component of $W_{1}-K_{1}$ containing $w$. Observe that $V \cap K \subset K$ $-K_{1}$ is a relatively closed 0 -dimensional subset of $V$ and, hence, $V \cap C_{+}=V-K$ is a dense, connected, open subset of $V$.

Let $A$ be an arc with $\partial A \subset K_{1}$ and $A-\partial A \subset V \cap C_{+}$. Split $A$ into subarcs $A_{1}, A_{2}$ having just an endpoint $e$ in common. Then $e \in V \cap C_{+}$. An argument like that in Lemma 2.5 verifies that $\check{H}^{n+1}\left(p^{-1} A ; \mathbf{Z}\right) \cong \mathbf{Z}$. The diagram

$$
\begin{gathered}
H_{1}\left(p^{-1} W_{1}\right) \stackrel{\gamma}{\rightarrow} H_{1}\left(p^{-1} W_{1}, p^{-1} W_{1}-p^{-1} A\right) \rightarrow \tilde{H}_{0}\left(p^{-1} W_{1}-p^{-1} A\right) \rightarrow 0 \\
\downarrow \cong \\
\check{H}^{n+1}\left(p^{-1} A\right) \cong \mathbf{Z}
\end{gathered}
$$


indicates that $p^{-1} A$ separates $p^{-1} W_{1}$ (recall the previous argument in the proof of Lemma 2.3 that $\gamma$ is trivial). Similarly, $p^{-1} A$ separates $p^{-1} U_{0}$. Hence, $A$ separates both $W_{1}$ and $U_{0}$.

Our initial specification that $H_{n+1}\left(p^{-1} W_{1}\right) \rightarrow H_{n+1}\left(p^{-1} U_{0}\right)$ be trivial starts the chase through the diagram

$$
\begin{aligned}
& H_{n+1}\left(p^{-1} U_{0}\right) \stackrel{\cong}{\rightarrow} H^{1}\left(\mathrm{Cl} p^{-1} U_{0}, \operatorname{Fr} p^{-1} U_{0}\right) \\
& \uparrow \\
& H^{1}\left(\mathrm{Cl}^{-1} U_{0}, \mathrm{Cl}^{-1} U_{0}-p^{-1} W_{1}\right) \\
& H_{n+1}\left(p^{-1} W_{1}\right) \stackrel{\cong}{\rightarrow} H^{1}\left(\mathrm{Cl} p^{-1} W_{1}, \operatorname{Fr} p^{-1} W_{1}\right)
\end{aligned}
$$

leading to the detection that $j$ is trivial. (The isomorphisms on the left arise from Lefshetz Duality [S, p. 297] while that on the right is an excision.) Since $j$ is trivial, the diagram

$$
\begin{array}{ccccc}
0 & \rightarrow & \tilde{H}^{0}\left(\operatorname{Fr} p^{-1} U_{0}\right) & \rightarrow & \check{H}^{1}\left(\mathrm{Cl}^{-1} U_{0}, \operatorname{Fr} p^{-1} U_{0}\right) \\
& & & & \\
0 & \rightarrow & \tilde{\check{H}}^{0}\left(\mathrm{Cl} p^{-1} U_{0}-p^{-1} W_{1}\right) & \rightarrow & H^{1}\left(\mathrm{Cl}^{-1} U_{0}, \mathrm{Cl}^{-1} U_{0}-p^{-1} W_{1}\right)
\end{array}
$$

can be used to detect that $i$ is trivial and, consequently, $\operatorname{Fr} p^{-1} U_{0}$ is contained in a single component of $\left(\mathrm{Cl}^{-1} U_{0}\right)-p^{-1} W_{1}$. Thus, Fr $p^{-1} U_{0}$ is contained in a single component of $\left(\mathrm{Cl}^{-1} U_{0}\right)-p^{-1} A$. This shows that one component of $\left(\mathrm{Cl}^{-1} U_{0}\right)-$ $p^{-1} A$ is also a component of $M-p^{-1} A$, as well as that one component of $B-A$ is a subset of $U_{0}$. This completes the proof of Lemma 3.4.

It remains to analyze the set $W_{1} \cap \operatorname{Fr} V$ with the aim of determining that it totally disconnected. Thereby we shall discover that $V=W_{1}$ and that $W_{1} \cap K_{1}$ is a nonempty ( $b_{0} \in W_{1} \cap K_{1}$ ), totally disconnected subset of $K_{1}$.

\section{LEMMA 3.5. $W_{1} \cap$ Fr $V$ contains no arc.}

Proof. Suppose that $A \subset W_{1} \cap \operatorname{Fr} V$ is an arc. Recall that the choice of $W$ and $b_{0}$ was based on the stipulation that the shape retraction $\underline{r}: p^{-1} W \rightarrow p^{-1} b_{0}$ restricts to a shape equivalence from $p^{-1} b$ to $p^{-1} b_{0}$, for each $b \in K_{1} \cap W$. It follows that, for each subarc $A^{\prime}$ of $A$ and $x \in A^{\prime}$, the inclusion $p^{-1} x \rightarrow p^{-1} A^{\prime}$ is a shape equivalence. Consequently, we can choose connected open sets $P$ and $N$, with $P \subset N \subset W_{1}$, such that

$$
N \cap A=A-\partial A=P \cap A,
$$

the inclusion induces an injection of $\underline{\pi}_{1}\left(p^{-1}(A-\partial A)\right)$ to $\pi_{1}\left(p^{-1} N\right)$, and

$$
\operatorname{Im}\left\{\underline{\pi}_{1}\left(p^{-1}(A-\partial A)\right) \rightarrow \pi_{1}\left(p^{-1} N\right)\right\}=\operatorname{Im}\left\{\pi_{1}\left(p^{-1} P\right) \rightarrow \pi_{1}\left(p^{-1} N\right)\right\} .
$$

Claim. The set $p^{-1}(A-\partial A)$ separates $p^{-1} P$ into precisely two connected pieces. Furthermore, if $A^{\prime}$ is a subarc of $A-\partial A$ and if $P^{\prime} \subset N^{\prime} \subset P$ are connected open sets chosen so that $N^{\prime} \cap A^{\prime}=A^{\prime}-\partial A^{\prime}=P^{\prime} \cap A^{\prime}$, inclusion induces an injection of $\pi_{1}\left(p^{-1}\left(A^{\prime}-A^{\prime}\right)\right)$ to $\pi_{1}\left(p^{-1} N^{\prime}\right)$, and

$$
\operatorname{Im}\left\{\underline{\pi}_{1}\left(p^{-1}\left(A^{\prime}-\partial A^{\prime}\right)\right) \rightarrow \pi_{1}\left(p^{-1} N^{\prime}\right)\right\}=\operatorname{Im}\left\{\pi_{1}\left(p^{-1} P^{\prime}\right) \rightarrow \pi_{1}\left(p^{-1} N\right)\right\},
$$


then distinct components of $p^{-1} P^{\prime}-p^{-1}\left(A^{\prime}-\partial A^{\prime}\right)$ are contained in distinct components of $p^{-1} P-p^{-1}(A-\partial A)$.

Before proving this, we use it to arrive at the contradiction that establishes Lemma 3.5. Transferring the conclusions via the map $p$, we have that $P-A=Z_{1} \cup Z_{2}$, where $Z_{1}$ and $Z_{2}$ are distinct components. An easy consequence of the "furthermore" in the Claim is that every point of $A-\partial A$ is arcwise accessible from each of $Z_{1}$ and $Z_{2}$. As one of $Z_{1}$ or $Z_{2}$ meets $V$, say $Z_{1} \cap V \neq \varnothing$, there is a 4-od $T$ (i.e., $T$ is the cone on a four-point set) with $T \subset\left(Z_{1} \cap C_{+}\right) \cup(A-\partial A)$, with the cone point in $V$ and with $T \cap(A-\partial A)$ equal to the four endpoints of $T$. Let $T^{\prime}$ denote the subset of $T$ that is the "sub-4-od" whose endpoints lie in Fr $V$ but is otherwise contained in $V \cap C_{+}$. Name the endpoints of $T$ to be $a_{1}, a_{2}, a_{3}, a_{4}$ and the corresponding endpoints of $T^{\prime}$ to be $a_{1}^{\prime}, a_{2}^{\prime}, a_{3}^{\prime}, a_{4}^{\prime}$. Let $\langle x, y\rangle$ denote the subarc of $T$ connecting any given pair of points $x, y \in T$. Lemma 3.4 implies that for some pair, say $a_{1}^{\prime}, a_{2}^{\prime}$, $p^{-1}\left(\left\langle a_{1}^{\prime}, a_{2}^{\prime}\right\rangle\right)$ separates $p^{-1} a_{3}^{\prime}$ from $p^{-1} a_{4}^{\prime}$ and, therefore, that $\left\langle a_{1}^{\prime}, a_{2}^{\prime}\right\rangle$ separates $a_{3}^{\prime}$ from $a_{4}^{\prime}$. This is an impossibility, since $\left\langle a_{3}^{\prime}, a_{3}\right\rangle \cup Z_{2} \cup\left\langle a_{4}^{\prime}, a_{4}\right\rangle$ is a connected set $\operatorname{missing}\left\langle a_{1}^{\prime}, a_{2}^{\prime}\right\rangle$.

Proof of the Claim. Since $p^{-1} x$ and $p^{-1} y$ are "connected" by a shape equivalence for $x, y \in A$, an argument like the one given for Lemma 2.2 shows that inclusion induces an isomorphism $\check{H}^{n}\left(p^{-1} A^{\prime}\right) \rightarrow \check{H}^{n}\left(p^{-1} a\right)$ and that $\check{H}^{n+1}\left(p^{-1} A^{\prime}\right)$ $=0$ for each subarc $A^{\prime}$ of $A$ and each $a \in A^{\prime}$. The exact cohomology sequence for the pair $\left(p^{-1} A^{\prime}, p^{-1} \partial A^{\prime}\right)$ and properties of cohomology with compact supports [S, p. 321] give

$$
\check{H}_{c}^{n+1}\left(p^{-1}\left(A^{\prime}-\partial A^{\prime}\right)\right) \cong \check{H}^{n+1}\left(p^{-1} A^{\prime}, p^{-1} \partial A^{\prime}\right)=0
$$

and a comparison of two such sequences reveals that inclusion induces an isomorphism

$$
H_{c}^{n+1}\left(p^{-1}\left(A^{\prime}-\partial A^{\prime}\right)\right) \rightarrow H_{c}^{n+1}\left(p^{-1}\left(A^{\prime \prime}-\partial A^{\prime \prime}\right)\right)
$$

for subarcs $A^{\prime \prime} \subset A^{\prime}$ of $A$. The diagram

$$
\begin{array}{ccc}
H_{1}\left(p^{-1} N\right) & \stackrel{\beta}{\rightarrow} & H_{1}\left(p^{-1} N, p^{-1}(N-A)\right) \\
\uparrow \phi & \uparrow \cong \\
H_{1}\left(p^{-1} P\right) & \stackrel{\gamma}{\rightarrow} & H_{1}\left(p^{-1} P, p^{-1}(P-A)\right) \\
& \uparrow \cong & \rightarrow \\
& \\
& & H_{c}^{n+1}\left(p^{-1}(A-\partial A)\right) \cong Z
\end{array}
$$

can be used to establish the Claim, provided $\gamma$ is the trivial homomorphism. [The "furthermore" follows by comparing the diagrams for $A \subset P \subset N$ and $A^{\prime} \subset P^{\prime} \subset N^{\prime}$ and by using the inclusion-induced isomorphism

$$
\left.H_{c}^{n+1}\left(p^{-1}(A-\partial A)\right) \rightarrow H_{c}^{n+1}\left(p^{-1}\left(A^{\prime}-\partial A^{\prime}\right)\right) .\right]
$$

The triviality of $\gamma$ is immediate if $\underline{\pi}_{1}\left(p^{-1} a\right)=0$ for some (hence, any) $a \in A$, as the homomorphism $\phi$ is then trivial. An outline of the argument that $\gamma$ is trivial in 
general will follow; this will be done by showing $\beta(2 \phi(e))=0$ for each $e \in$ $H_{1}\left(p^{-1} P\right)$ and, hence, as $\beta$ is a homomorphism to $\mathbf{Z}, \beta \phi$ is trivial. Of course, it suffices to show that $2 \phi(e)$ is an element of

$$
\operatorname{Im}\left\{H_{1}\left(p^{-1}(N-A)\right) \rightarrow H_{1}\left(p^{-1} N\right)\right\} .
$$

In fact, we show that in the diagram

$$
\begin{aligned}
& \pi_{1}\left(p^{-1}(N-A), x_{0}\right) \stackrel{\delta}{\rightarrow} \pi_{1}\left(p^{-1} N, x_{0}\right) \\
& \begin{array}{c}
\downarrow \eta \\
\pi_{1}\left(p^{-1} P, x_{0}\right)
\end{array}
\end{aligned}
$$

$2 \eta(e) \in \operatorname{Im} \delta$ for each $e \in \pi_{1}\left(p^{-1} P, x_{0}\right)$, where the basepoint $x_{0}$ lies in $p^{-1}(P-A)$.

Let $f: \tilde{N} \rightarrow p^{-1} N$ denote the universal covering space. Choose $y_{0} \in f^{-1}\left(x_{0}\right)$. Let $\tilde{P}_{0}$ denote the component of $f^{-1}(P)$ containing $y_{0}$ and let

$$
\tilde{A}_{0}=\tilde{P}_{0} \cap f^{-1} p^{-1}(A-\partial A) .
$$

It follows from the $\pi_{1}$ conditions stipulated prior to the statement of the Claim that $\tilde{A}_{0}$ is the only component of $f^{-1} p^{-1}(A-\partial A)$ in $\tilde{P}_{0}$ and that $\underline{\pi}_{1}\left(\tilde{A}_{0}\right)$ is trivial. A computation outlined below establishes $H_{c}^{n+1}(\tilde{A}) \cong \mathbf{Z}$, and then the simplyconnected case of this result applies to reveal $\tilde{P}_{0}-\tilde{A}_{0}=C_{1} \cup C_{2}$, where $C_{1}$ and $C_{2}$ are distinct components. For an arbitrary representative $e:(I,\{0,1\}) \rightarrow\left(p^{-1} P, x_{0}\right)$ of an element (also called) $e$ of $\pi_{1}\left(p^{-1} P, x_{0}\right)$, if there is a lift $\tilde{e}: I \rightarrow \tilde{P}_{0}$ of $e$ with $\tilde{e}(0)$ and $\tilde{e}(1)$ contained in the same $C_{i}$, then the loop obtained by connecting $\tilde{e}(0)$ and $\tilde{e}(1)$ via a path in that $C_{i}$ is necessarily null homotopic in $\tilde{N}$ and, consequently, $e$ is homotopic rel $\{0,1\}$ in $p^{-1} N$ to $e^{\prime}:(I,\{0,1\}) \rightarrow\left(f\left(C_{i}\right), x_{0}\right)$. As $f\left(C_{i}\right) \cap p^{-1} A=\varnothing$, the element $\eta(e)=\eta\left(e^{\prime}\right)$ is in $\operatorname{Im} \delta$. If no lift $\tilde{e}$ to $\tilde{P}_{0}$ has $\tilde{e}(0)$ and $\tilde{e}(1)$ contained in the same $C_{i}$, then each lift of $2 e$ to $\tilde{P}_{0}$ must have this property. Thus, in either case, $\eta(2 e) \in \operatorname{Im} \delta$.

Finally, to finish off the Claim, we describe the computation of $H_{c}^{n+1}\left(\tilde{A_{0}}\right)$. Set $\tilde{E}$ equal to that component of $f^{-1} p^{-1} E$ contained in $\tilde{A}_{0}$, where $E$ is a closed subarc of $A$. Given any point $a_{0} \in E$, we specify a shape equivalence $s$ from $p^{-1} a_{0}$ to a closed orientable $n$-manifold $g_{0}$ and a shape deformation retraction $\underline{r}^{\prime}: p^{-1} E \rightarrow p^{-1} a_{0}$. Denote by $\tilde{g}_{0}$ the universal cover of $g_{0}$ and by $\tilde{s}: \tilde{E} \cap f^{-1} p^{-1} a_{0} \rightarrow \tilde{g}_{0}$ and $\underline{\tilde{r}}^{\prime}$ : $\tilde{E} \rightarrow \tilde{E} \cap f^{-1} p^{-1} a_{0}$ lifts of $s$ and $\underline{r}^{\prime}$, respectively. Computing with $\tilde{s}$ and $\underline{\tilde{r}}^{\prime}$ establishes that $H_{c}^{n}\left(E \cap f^{-1} p^{-1} a_{0}\right) \cong \mathbf{Z}$, that $\check{H}_{c}^{n+1}(\tilde{E})=0$, and that inclusion induces an isomorphism

$$
\check{H}_{c}^{n}(\tilde{E}) \rightarrow \check{H}_{c}^{n}\left(\tilde{E} \cap f^{-1} p^{-1} a_{0}\right) .
$$

The exact cohomology sequence (with compact supports) of the pair $(\tilde{E}, \tilde{E} \cap$ $f^{-1} p^{-1} \partial E$ ) displays that

$$
\check{H}_{c}^{n+1}\left(\tilde{E}, \tilde{E} \cap f^{-1} p^{-1} \partial E\right) \cong \check{H}_{c}^{n+1}\left(\tilde{E}-f^{-1} p^{-1} \partial E\right) \cong \mathbf{Z} .
$$

For a pair of closed subarcs $E \subset F$ of $A$, a comparison of the computation just described for each reveals that inclusion induces an isomorphism

$$
\check{H}_{c}^{n+1}\left(\tilde{E}-f^{-1} p^{-1} \partial E\right) \cong \check{H}_{c}^{n+1}\left(\tilde{F}-f^{-1} p^{-1} \partial F\right) .
$$


(Specifically, compare the pair $\left(\tilde{F}, \tilde{F} \cap f^{-1} p^{-1} \partial F\right)$ and the pair $\left(\tilde{F}, \tilde{F} \cap f^{-1} p^{-1}\right.$ $\mathrm{Cl}(F-E))$ to determine that

$$
\check{H}_{c}^{n+1}\left(\tilde{F}, \tilde{F} \cap f^{-1} p^{-1} \partial F\right) \cong \check{H}_{c}^{n+1}\left(\tilde{F}, \hat{F} \cap f^{-1} p^{-1} \mathrm{Cl}(F-E)\right)
$$

and, then, use the excision axiom to deduce that the latter group is isomorphic to

$$
\left.\check{H}_{c}^{n+1}\left(\tilde{E}-f^{-1} p^{-1} \partial E\right) .\right)
$$

Finally, $\check{H}_{c}^{n+1}\left(\tilde{A_{0}}\right)$ is the direct limit of the groups $\check{H}_{c}^{n+1}\left(\tilde{E}-f^{-1} p^{-1} \partial E\right)$ where $E$ ranges over closed subarcs of $A$ and, hence, $\check{H}_{c}^{n+1}\left(A_{0}\right) \cong \mathbf{Z}$.

Proof of Proposition 3.2. Since $W_{1} \cap \operatorname{Fr} V$ contains no arc, no compact connected subset of it, other than a singleton, can be locally connected. Consequently, we can show $W_{1} \cap$ Fr $V$ to be totally disconnected by proving it contains no sequence $Z_{1}, Z_{2}, \ldots$ of pairwise disjoint, compact connected subsets, each with at least two points, that converges in the Hausdorff metric to another compact connected subset $Z$, also with at least two points. The possibility that such a sequence might exist is eliminated by considering the following two cases.

First, $Z$ contains four points $a_{1}, a_{2}, a_{3}, a_{4}$ which are arcwise accessible from $V$. Then there exists a 4-od $T$ in $\left(V \cap C_{+}\right) \cup Z$, with $T \cap Z=\left\{a_{1}, a_{2}, a_{3}, a_{4}\right\}$. Lemma 3.4 implies that for some pair, say $\left\{a_{1}, a_{2}\right\}$, the subarc $\left\langle a_{1}, a_{2}\right\rangle$ of $T$ separates $a_{3}$ from $a_{4}$ in $V$. This is not possible because the connected sets $Z_{1}, Z_{2}, \ldots$ miss $T$ but have both $a_{3}$ and $a_{4}$ as limit points.

Second, the set of points in $Z$ that are arcwise accessible from $V$ is not dense in $Z$. In this case, there exists a 4-od $T$ in $\left(V \cap C_{+}\right) \cup\left(W_{1} \cap \operatorname{Fr} V\right)$, having its endpoints $a_{1}, \ldots, a_{4}$ in $Z$ and containing a "sub-4-od" $T$ ', which, in turn, has its endpoints $a_{1}^{\prime}, \ldots, a_{4}^{\prime}$ in $W_{1} \cap \operatorname{Fr} V$, disjoint from $\left\{a_{1}, \ldots, a_{4}\right\}$, and satisfying $T^{\prime}-\left\{a_{1}^{\prime}, \ldots, a_{4}^{\prime}\right\}$ $\subset V \cap C_{+}$. Lemma 3.4 again implies that for some pair, say $\left\{a_{1}^{\prime}, a_{2}^{\prime}\right\}$, the subarc $\left\langle a_{1}^{\prime}, a_{2}^{\prime}\right\rangle$ of $T^{\prime}$ separates $a_{3}^{\prime}$ from $a_{4}^{\prime}$. This is not possible because $\left\langle a_{3}^{\prime}, a_{3}\right\rangle \cup Z$ $\cup\left\langle a_{4}^{\prime}, a_{4}\right\rangle$ is a connected subset of $B$ missing $\left\langle a_{1}^{\prime}, a_{2}^{\prime}\right\rangle$ but containing both $a_{3}^{\prime}$ and $a_{4}^{\prime}$.

Lemma 3.1 and Corollary 3.3 combine to form our main result.

THEOREM 3.6. If $M$ is an orientable $(n+2)$-manifold and $G$ is a usc decomposition of $M$ into continua having the shape of closed, orientable n-manifolds, then $B=M / G$ is a 2-manifold (without boundary).

Orientability of the elements of $G$ is a fundamental hypothesis in Theorem 3.6; orientability of the source manifold $M$ is less crucial, as the next result demonstrates.

THEOREM 3.7. If $G$ is a usc decomposition of an $(n+2)$-manifold $M$ into continua having the shape of closed, orientable $n$-manifolds, then $B=M / G$ is a 2-manifold possibly with boundary.

Proof. Assume $M$ to be nonorientable. Consider the orientable double covering $\theta: \tilde{M} \rightarrow M$ and usc decomposition $\tilde{G}$ into the components of $\theta^{-1}(g), g \in G$. The 
nontrivial covering homeomorphism $h: \tilde{M} \rightarrow \tilde{M}$, which respects elements of $\tilde{G}$, induces an involution $u$ on $\tilde{M} / \tilde{G}$, and the diagram

$$
\begin{array}{ccc}
\tilde{M} & \stackrel{\theta}{\rightarrow} & M \\
\tilde{p} \downarrow & & p \downarrow \\
\tilde{M} / \tilde{G} & \stackrel{T}{\rightarrow} & M / G
\end{array}
$$

induces a map $T: \tilde{M} / \tilde{G} \rightarrow M / G$ behaving just like the map of $\tilde{M} / \tilde{G}$ to the orbit space of $u$. It is well known [Wh] that the orbit space of every involution on a 2-manifold is a 2-manifold with boundary (the boundary may be empty).

Before closing down our analysis of the troublesome spots $D$ and $K$, the discontinuity and degeneracy subsets of $B$, we provide one last analysis.

Proposition 3.8. Assume $M$ (orientable) satisfies $H_{1}(M)=0$ and $K \neq \varnothing$. Then $K \cup D$ consists of exactly one point.

Proof. Find an $\operatorname{arc} A$ in $B$ with $\partial A \subset K \cup D, \partial A \cap K \neq \varnothing$, and $A-\partial A \subset C_{+}$. If $\partial A \subset K$, then as before $\breve{H}^{n+1}\left(p^{-1} A ; \mathbf{Z}\right) \cong \mathbf{Z}$; if $d \in \partial A \cap D$, then

$$
\check{H}^{n+1}\left(p^{-1} A ; \mathbf{Z} / k \mathbf{Z}\right) \cong \mathbf{Z} / k \mathbf{Z},
$$

where $\alpha_{d}(x)=k$ for all $x \neq d$ sufficiently close to $d$. As a result, with the appropriate coefficients the diagram

$$
\begin{aligned}
& 0=H_{1}(M) \rightarrow H_{1}\left(M, M-p^{-1} A\right) \rightarrow \tilde{H}_{0}\left(M-p^{-1} A\right) \rightarrow \tilde{H}_{0}(M)=0 \\
& \downarrow \cong \\
& \check{H}^{n+1}\left(p^{-1} A\right)
\end{aligned}
$$

reveals $\tilde{H}_{0}\left(M-p^{-1} A\right)$ to be nontrivial, implying that the arc $A$ separates the 2-manifold $B$, an impossibility.

As applications of Theorem 3.6, we mention some structural limitations upon the manifolds $M$ admitting decompositions into codimension-two submanifolds.

Proposition 3.9. Suppose $M$ is a connected $(n+2)$-manifold with at least two ends and with $H_{1}(M ; Z / 2 Z)=0$. Then $M$ admits no usc decomposition $G$ into continua having the shape of closed, orientable n-manifolds.

Proof. If it did, the (noncompact) decomposition space $B$ would satisfy $H_{1}(B ; \mathbf{Z} / 2 \mathbf{Z})=0[\mathrm{~B}]$, indicating that $B$ is the plane. Here the key property is that $B$ has one end. The desired contradiction stems from the fact that proper, monotone maps preserve the number of ends.

Proposition 3.10. Suppose $M$ is a noncompact, connected $(n+2)$-manifold such that for each compact subset $C$ of $M$ there exists another compact set $C^{\prime} \supset C$ such that

$$
H_{1}\left(M-C^{\prime} ; \mathbf{Z} / 2 \mathbf{Z}\right) \rightarrow H_{1}(M-C ; \mathbf{Z} / 2 \mathbf{Z})
$$

is trivial. Then $M$ admits no usc decomposition $G$ into continua having the shape of closed, orientable n-manifolds. 
Proof. Suppose otherwise. Then $B=M / G$ is a noncompact 2-manifold for which $H_{1}(B ; \mathbf{Z} / 2 \mathbf{Z})$ is trivial at infinity, by $[\mathbf{B}]$ and the hypothesis. This is impossible: every noncompact 2-manifold contains loops arbitrarily close to infinity that fail to be null homologous in the complement of a given compactum.

Our chief interest was in determining whether $E^{n+2}$ admits a decomposition ino codimension-two submanifolds, which now is settled.

COROLlaRY 3.11. There is no usc decomposition of $E^{n+2}$ into continua having the shape of closed, orientable n-manifolds.

Remark. The upper semicontinuity of the decompositions $G$ has been fundamental to the discussion throughout this paper. Reinforcing this point, a remarkably explicit partition of $E^{3}$ into (round) circles is given in [Sz].

Finally, in the compact case, we have

Proposition 3.12. Suppose $M^{n+2}$ is a closed, orientable n-manifold for which $H_{2}(M ; \mathbf{Z} / 2 \mathbf{Z})=0$. Then $M$ admits no usc decomposition $G$ into continua having the shape of closed n-manifolds with $\check{H}_{1}(g ; \mathbf{Z} / 2 \mathbf{Z})=0$ for all $g \in G$.

Proof. If it did, $B$ would be a closed 2-manifold, and $H_{2}(B ; \mathbf{Z} / 2 \mathbf{Z}) \neq 0$. Under these hypotheses, the Vietoris-Begle Theorem [B] ensures that

$$
p_{*}: H_{2}(M ; \mathbf{Z} / 2 \mathbf{Z}) \rightarrow H_{2}(B ; \mathbf{Z} / 2 \mathbf{Z})
$$

is surjective, a contradiction.

4. Structure of the sheaf $\mathscr{H}^{n}$. We shall denote by $\mathscr{H}^{n}$ the presheaf on $B$ determined by setting $\mathscr{H}^{n}(U)=H^{n}\left(p^{-1} U ; \mathbf{Z}\right)$ (and using inclusion induced homomorphisms) as well as the associated sheaf, whose stalk at a point $x \in B$ is $\check{H}^{n}\left(p^{-1} x ; \mathbf{Z}\right)$. The path leading to the conclusion that $B$ is a 2 -manifold also brought us to a determination that $\mathscr{H}^{n}$ is locally constant except at points of the sparse set $K \cup D_{+}$. A final refinement is

THEOREM 4.1. Suppose $M$ is an orientable $(n+2)$-manifold and $G$ is a usc decomposition of $M$ into continua having the shape of closed, orientable n-manifolds. Then the decomposition space $B$ is a 2-manifold and B contains a locally finite (closed) subset $F$ such that $\mathscr{H}^{n}$ is locally constant at each point of $B-F$. Furthermore, if $\check{H}_{1}(g ; \mathbf{Z}) \cong 0$ for each $g \in G$, then $F=\varnothing$.

Proof. Since it is already established that $B$ is a 2-manifold and $\mathscr{H}^{n}$ is locally constant over $B \backslash K \cup D_{+}$, we need to show that $K \cup D_{+}$is locally finite and, under the additional constraints of the "furthermore", that it is empty.

The local finiteness of $K \cup D_{+}$is detected by precluding the existence of three possible types of convergent sequences from $K \cup D_{+}$.

First, suppose there is a sequence $\left\{x_{i}\right\} \subset K$ with $x_{i} \rightarrow x \in K$. It had been previously observed that fact (1) detects that the local winding function at any point of $K$ is zero at some point of the continuity set $C_{+}$. As we currently know that $U \cap C_{+}$is connected whenever $U$ is an open connected set, each such local winding function is zero at every point of $C_{+}$in its domain. Thus, the argument in Lemma 
3.4 would show that an $\operatorname{arc} A$ connecting $x_{i}$ and $x_{i+1}$, where $i$ is chosen so that $A$ is contained in an appropriately small neighborhood of $x$ and $A-\partial A \subset C_{+}$, would separate a connected neighborhood of $x$. This clearly is not possible as $B$ is a 2-manifold (without boundary).

Second, suppose that some point $x \in D_{+}$is a limit point of $D_{+}-x$. Let $\alpha$ denote the local winding function at $x$ defined on a connected neighborhood $U$ of $x$ and, for $d \in D_{+} \cap U$, let $\alpha_{d}$ denote the local winding function of $d$ defined on a connected neighborhood $V_{d} \subset U$ of $d$. Since $U \cap C_{+}$is connected, $\alpha$ is constant on $U \cap C_{+}$, say $\alpha(b)=r$. Similarly, $\alpha_{d}$ is constant on $V_{d} \cap C_{+}$, say $\alpha_{d}(b)=r_{d}$. According to fact (1), $r=k \cdot r_{d}$ for $b \in V_{d} \cap C_{+}$and, consequently, the integers $r_{d}$ are bounded (by $r$ ). Of course, $r_{d}>1$ as $d \in D_{+}$. Thus, we can specify a sequence $\left\{x_{i}\right\} \subset D_{+}-x$ with $x_{i} \rightarrow x$ and with the local winding functions at the $x_{i}$ 's taking on the single value $q>1$ at points in the continuity set $C_{+}$. An argument as in Lemma 3.4 using $\mathbf{Z} / q \mathbf{Z}$-coefficients would lead to the contradiction that an $\operatorname{arc} A$, chosen as in the preceding paragraph, separates a connected neighborhood of $x$.

Third, suppose that there is a sequence $\left\{x_{i}\right\} \subset D_{+}$with $x_{i} \rightarrow x \in K$. As before, we assume that the $x_{i}$ 's are contained in a neighborhood $U$ of $x$ on which the local winding function $\alpha$ at $x$ is defined. Specify connected neighborhoods $V_{i} \subset U$ of $x_{i}$ on which the local winding function $\alpha_{i}$ at $x_{i}$ is defined and set $r_{i}$ equal to the constant value of $\alpha_{i}$ on $V_{i} \cap C_{+}$. In contrast with the previous situation, we cannot conclude that the $r_{i}$ 's are bounded, as $\alpha(b)=0$ for $b \in U_{0} \cap C_{+}$. Consider an arc $A$ connecting $x$ and $x_{i}$ where $A$ is chosen in a small neighborhood of $x$ containing $x_{i}$ and where $A-\partial A \subset C$. An argument as in Lemma 3.4 using $\mathbf{Z} / r_{i} \mathbf{Z}$-coefficients produces the contradiction that $A$ separates a connected neighborhood of $x$ provided the homomorphism $H_{1}\left(p^{-1} W_{1}\right) \rightarrow H_{1}\left(p^{-1} W_{1}, p^{-1} W_{1}-p^{-1} A\right)$ is trivial $\left(W_{1}\right.$ being chosen near the start of the proof at Lemma 3.4). In the preceding two paragraphs, the fact that $x \notin A$ facilitates the computation. In the current situation, we must insist that, after choices of neighborhoods $W_{1} \subset U_{0} \subset U$ of $x$ are made, $i$ is chosen with $p^{-1} A$ so close to $p^{-1} x$ that

$$
\operatorname{Im}\left\{\check{H}_{1}\left(p^{-1} x\right) \rightarrow H_{1}\left(p^{-1} U\right)\right\}=\operatorname{Im}\left\{H_{1}\left(p^{-1}\left(U_{0}-A\right)\right) \rightarrow H_{1}\left(p^{-1} U\right)\right\} .
$$

This poses no difficulties since generators for $\operatorname{Im}\left\{\check{H}_{1}\left(p^{-1} x\right) \rightarrow H_{1}\left(p^{-1} U\right)\right\}$ can be found in $H_{1}\left(p^{-1}\left(U_{0}-x\right)\right)$.

We now assume that $\breve{H}_{1}\left(p^{-1} x ; \mathbf{Z}\right) \cong 0$ for each $x \in B$. Suppose, contrary to the Claim of the "furthermore", that $b \in K \cup D_{+}$. Specify an open 2-cell $\Delta \subset B$ with $\Delta \cap\left(K \cup D_{+}\right)=\{b\}$ so that there is a shape strong deformation retraction $r$ : $p^{-1} \Delta \rightarrow p^{-1} b$, the deformation occurring in a neighborhood of $p^{-1} b$. Set $\Delta^{*}=\Delta-$ $\{b\}$ and choose any $c \in \Delta^{*}$. Consider the diagram

$$
\begin{aligned}
& H^{n}\left(p^{-1} \Delta\right) \stackrel{\alpha}{\rightarrow} H^{n}\left(p^{-1} \Delta^{*}\right) \quad \rightarrow \quad H^{n+1}\left(p^{-1} \Delta, p^{-1} \Delta^{*}\right) \\
& \downarrow j^{*} \quad \downarrow i^{*} \\
& \check{H}^{n}\left(p^{-1} b\right) \stackrel{\phi}{\rightarrow} \quad \check{H}^{n}\left(p^{-1} c\right)
\end{aligned}
$$

where the top row is part of the exact sequence of the pair $\left(p^{-1} \Delta, p^{-1} \Delta^{*}\right), \phi$ is induced by the restriction of $\underline{r}$, and the remaining homomorphisms are induced by 
inclusions. In particular, $\phi j^{*}=i^{*} \alpha$. The existence of $\underline{r}$ assures that $j^{*}$ is into. The assumption that $\check{H}_{1}\left(p^{-1} b\right) \cong 0$ yields, by duality $[\mathbf{S}$, p. 296] that $H^{n+1}\left(p^{-1} \Delta, p^{-1} \Delta^{*}\right) \cong 0$. Consequently, the contradiction that $\phi$ is onto (and hence an isomorphism) will be reached once $i *$ is shown to be onto.

The universal coefficient theorem [S, p. 243] yields the short exact sequence

$$
\begin{aligned}
0 & \rightarrow \operatorname{Ext}\left(H_{n}\left(p^{-1} \Delta, p^{-1} \Delta^{*}\right), \mathbf{Z}\right) \rightarrow H^{n+1}\left(p^{-1} \Delta, p^{-1} \Delta^{*}\right) \\
& \rightarrow \operatorname{Hom}\left(H_{n+1}\left(p^{-1} \Delta, p^{-1} \Delta^{*}\right), \mathbf{Z}\right) \rightarrow 0
\end{aligned}
$$

and duality [S, p. 296] produces

$$
H_{n}\left(p^{-1} \Delta, p^{-1} \Delta^{*}\right) \cong \check{H}^{2}\left(p^{-1} b\right) \text { and } H_{n+1}\left(p^{-1} \Delta, p^{-1} \Delta^{*}\right) \cong \check{H}^{1}\left(p^{-1} b\right) .
$$

The same exact sequence applied to $\check{H}^{1}\left(p^{-1} b\right)$ reveals that $\check{H}^{1}\left(p^{-1} b\right) \cong 0$ and, then, to $\check{H}^{2}\left(p^{-1} b\right)$ reveals that $\check{H}^{2}\left(p^{-1} b\right)$ is free. It follows that $H^{n+1}\left(p^{-1} \Delta, p^{-1} \Delta^{*}\right) \cong 0$.

We begin the computation of $i{ }^{*}$ 's surjectiveness by expressing $\Delta-b=\Delta^{*}$ as the union of two open disks $R_{1}$ and $R_{2}$ whose intersection is a pair of open disks $V_{1}$ and $V_{2}$ with $c \in V_{1}$. Further, we specify a point $d \in V_{2}$ and $\operatorname{arcs} A_{1} \subset R_{1}$ and $A_{2} \subset R_{2}$ connecting $e$ and $d$. The strategy is to decompose $i^{*}$ as

$$
H^{n}\left(p^{-1} \Delta^{*}\right) \underset{\delta}{\rightarrow} H^{n}\left(p^{-1}\left(A_{1} \cup A_{2}\right)\right) \underset{\beta}{\rightarrow} \check{H}^{n}\left(p^{-1} c\right),
$$

and then to determine that both inclusion induced homomorphisms $\delta$ and $\beta$ are surjective.

The Mayer-Vietoris sequence

$$
\begin{array}{ccc}
\check{H}^{n}\left(p^{-1}\left(A_{1} \cap A_{2}\right)\right) & \rightarrow & \check{H}^{n+1}\left(p^{-1}\left(A_{1} \cup A_{2}\right)\right) \rightarrow 0 \\
\downarrow \cong & \downarrow \cong \\
\check{H}^{n}\left(p^{-1} c\right) \oplus \check{H}^{n}\left(p^{-1} d\right) & \mathbf{Z} \\
\downarrow \cong & \\
\mathbf{Z} \oplus \mathbf{Z} &
\end{array}
$$

contains adequate information for discovering that $\beta$ is surjective. The computation of $\check{H}^{n+1}\left(p^{-1}\left(A_{1} \cup A_{2}\right)\right)$ is essentially done in Lemma 2.3 while the zero at the right is a consequence of Lemma 2.2. A further consequence of the latter is that, for $i=1,2$, there is an element $a_{i} \in \check{H}^{n}\left(p^{-1} A_{i}\right)$ that restricts to a generator in both $\check{H}^{n}\left(p^{-1} c\right)$ and $\check{H}^{n}\left(p^{-1} d\right)$. Hence, essentially either $\gamma\left(a_{1}, a_{2}\right)=(0,0)$ or $\gamma\left(a_{1}, a_{2}\right)=$ $(2,0)$. The latter would force $\breve{H}^{n+1}\left(p^{-1}\left(A_{1} \cup A_{2}\right)\right)$ to be a torsion group, which it is not, while the former readily shows that $\check{H}^{n}\left(p^{-1}\left(A_{1} \cup A_{1}\right)\right) \rightarrow \check{H}^{n}\left(p^{-1} A_{1}\right)$ is surjective, and, hence, $\beta$ is surjective.

Detecting the surjectivity of

$$
\check{H}^{n}\left(p^{-1} \Delta^{*}\right) \stackrel{\delta}{\rightarrow} \check{H}^{n}\left(p^{-1}\left(A_{1} \cup A_{2}\right)\right)
$$

is accomplished by comparing the spectral sequences of $p \mid: p^{-1} \Delta^{*} \rightarrow \Delta^{*}$ and $p \mid$ : $p^{-1}\left(A_{1} \cup A_{2}\right) \rightarrow A_{1} \cup A_{2}$. (The sequence is derived in [G] and is described adequately for our purpose in [DW2].) We only need to extract the commuting diagram

$$
\begin{array}{ccccc}
\check{H}^{n}\left(p^{-1} \Delta^{*} ; \mathbf{Z}\right) & \rightarrow & H^{0}\left(\Delta^{*} ; \mathscr{H}^{n}\right) & \rightarrow & 0 \\
\downarrow \delta & & \downarrow & & \\
\check{H}^{n}\left(p^{-1} A_{1} \cup A_{2} ; \mathbf{Z}\right) & \rightarrow & H^{0}\left(A_{1} \cup A_{2} ; \mathscr{H}^{n}\right) & \rightarrow & 0
\end{array}
$$


where the right-hand vertical homomorphism is inclusion induced. Furthermore, the homomorphism is an isomorphism since the early computation that

$$
\check{H}^{n+1}\left(p^{-1}\left(A_{1} \cup A_{2}\right) ; \mathbf{Z}\right) \cong \mathbf{Z}
$$

detects that the sheaf $\mathscr{H}^{n}$ is constant on $\Delta^{*}$. The surjectiviy of $\delta$ follows once we determine that the homomorphism $\eta$ is an isomorphism. From the spectral sequence of

$$
p \mid: p^{-1}\left(A_{1} \cup A_{2}\right) \rightarrow A_{1} \cup A_{2}
$$

we determine that kernel $\eta \cong H^{1}\left(A_{1} \cup A_{2} ; \mathscr{H}^{n-1}\right)$ but $\mathscr{H}^{n-1}$ is the zero-sheaf as each stalk $\check{H}^{n-1}\left(p^{-1} x ; \mathbf{Z}\right)$ is dually isomorphic to $\check{H}_{1}\left(p^{-1} x ; \mathbf{Z}\right) \cong 0$ (by assumption).

5. Examples. As a summary, we want to compare what is known about usc decompositions $G$ of orientable $(n+k)$-manifolds $M$ into connected, orientable $n$-manifolds in the two low-codimensional cases where $k=1$ and $k=2$. In either case $B=M / G$ is now known to be a boundaryless $k$-manifold.

Sometimes the decomposition map $p: M \rightarrow B$ can be employed to study the structure of $M$. This investigative tool is quite effective when $p$ is an approximate fibration. Unfortunately, that is not always the prevailing situation. Example 3 of [D] provides a decomposition $G$ of an $(n+1)$-manifold $M$ into homology $n$-spheres, some simply connected and others not; the decomposition map cannot be an approximate fibration because the decomposition elements have different shapes (different homotopy types). There is a similar decomposition of the $(n+2)$-manifold $M \times E^{1}$ into homology $n$-spheres.

When $k=1$ the structure of $M$ is exposed, to some extent, by one unifying feature: the elements of $G$ are all pairwise homologically equivalent [D, Corollary 6.3]. Consequently, the shape (neighborhood) retractions $V \rightarrow g$ induce homology isomorphisms $H_{j}\left(g^{\prime}\right) \rightarrow H_{j}(g)$ for all $g^{\prime}$ in $V$ sufficiently close to $g$. The first fact may appear to stem from the property that the continuity set $C_{+}$of $B$ coincides with $B$ itself. However, when $k=2$ the elements of $G$ need not all be homologically equivalent, not even if $C_{+}=B$.

EXAmPle. A usc decomposition $G$ of a connected, orientable $(n+2)$-manifold $M$ $(n \geqslant 2)$ into orientable $n$-manifolds such that $(1)$ in the resulting decomposition space $B$, each point belongs to the continuity set $C_{+}$, and (2) $G$ contains a pair of homologically inequivalent elements.

The crux of the matter is manifested when $n=2$, so focus on that case. Start with a knot $K$ in $S^{3}$, like the trefoil, whose closed complement fibers over $S^{1}$ with fiber $T$, where $T$ is a disk having a positive number of handles. Attach a (4-dimensional) 2-handle $H$ to $B^{4}$ along $U(K)$, a tubular neighborhood of $K$ in $S^{3}=\partial B^{4}$, via the framing determined by the longitude $\partial T$ of the knot space $\mathrm{Cl}\left(S^{3}-U(K)\right)$. The interior $M$ of the resulting 4-manifold with boundary represents the desired manifold.

The exceptional element $g_{0}$ of the decomposition $G$ yet to be specified is the 2-sphere in $M$ formed by the cone over $K$ from the center of $B^{4}$ and the core of the 
attached 2-handle, $h$. Topologically the other, more standard elements of $G$ all are the union $\hat{T}$ of $T$ and a 2-cell capping off $\partial T$. Each lies in $M$ as a copy of $\hat{T}$ in

$$
r \cdot\left(S^{3}-U(K)\right) \subset B^{4} \quad(\text { where } 0<r<1)
$$

concentric with one of the given fibers of $S^{3}-U(K)$, a copy of $B^{2}$ in

$$
B^{2} \times r \cdot \partial D^{2} \subset B^{2} \times D^{2}=h,
$$

and an annulus connecting the boundaries of the first two in the cone over $U(K)$ in $B^{4}$, as suggested by Figure 1 .

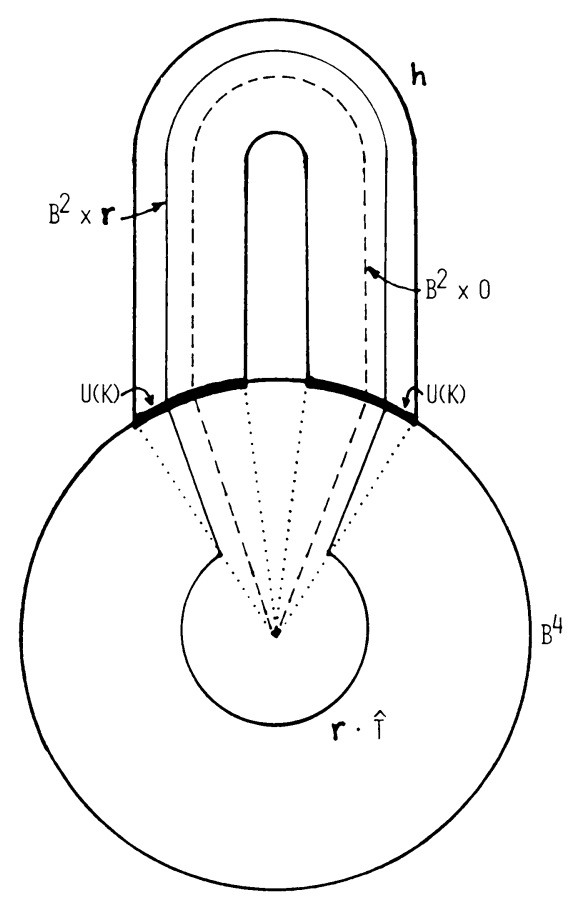

FIGURE 1

Away from the exceptional element $g_{0}$, the decomposition map behaves like the projection of $\hat{T} \times\left(E^{2}-0\right)$ to $E^{2}-0$. One can see that the winding function $\alpha_{b}$ is locally constant at $b=p\left(g_{0}\right)$ by observing the existence of a retraction $r_{0}: M \rightarrow g_{0}$ such that $r_{0}\left(M \cap B^{4}\right) \subset g_{0} \cap B^{4}$ and $r_{0} \mid h \cap M$ acts like the projection of $B^{2} \times$ Int $D^{2}$ to its core $B^{2} \times 0 \subset g_{0}$. For geometric reasons, $r_{0} \mid g^{\prime}: g^{\prime} \rightarrow g_{0}$ is a degree-one map, for each $g^{\prime} \in G[E]$. Obviously, for each $g \neq g_{0}, H_{1}\left(g^{\prime}\right)=H_{1}(\hat{T}) \not 0$.

\section{REFERENCES}

[B] E. (;. Begle. The Vietoris mapping theorem for bicompact spaces, Ann. of Math. (2) 51 (1950), $534-543$.

[CD1] D. S. Coram and P. F. Duvall, Mappings from $S^{3}$ to $S^{2}$ whose point inverses have the shape of $a$ circle. General Topology Appl. 10 (1979), 239-246.

[CI)2] . Finiteness theorems for approximate fibrations. Trans. Amer. Math. Soc. 269 (1982), $383-394$. 
[D] R. J. Daverman, Decompositions of manifolds into codimension one submanifolds, Compositio Math. (to appear).

[DH] R. J. Daverman and L. S. Husch, Decompositions and approximate fibrations, Michigan Math. J. 31 (1984), 197-214.

[DW1] R. J. Daverman and J. J. Walsh, Decompositions into codimension two spheres and approximate fibrations, Topology Appl., (to appear).

[DW2] _ Decompositions into submanifolds that yield generalized manifolds (in preparation).

[DS] J. Dydak and J. Segal, Local n-connectivity of decomposition spaces (to appear).

[E] D. B. A. Epstein, The degree of a map, Proc. London Math. Soc. (3) 16 (1966), 369-383.

[G] A. Grothendieck, Sur quelques points d'algèbre homologique, Tôhoku Math. J. (2) 9 (1957), 119-221.

[HW] W. Hurewicz and H. Wallman, Dimension theory, Princeton Univ. Press, Princeton, N. J., 1941.

[L] V.-T. Liem, Manifolds accepting codimension one sphere-like decompositions (to appear).

[S] E. H. Spanier, Algebraic Topologv, McGraw-Hill, New York, 1966.

[Sz] A. Szulkin, $R^{3}$ is the union of disjoint circles, Amer. Math. Monthly 90 (1964), 640-641.

[Wh] G. T. Whyburn, Interior transformations on surfaces, Amer. J. Math. 60 (1938), 477-490.

[Wi] R. L. Wilder, Topology of manifolds, Amer. Math. Soc. Colloq. Publ., vol. 32, Amer. Math. Soc., Providence, R. I., 1963.

Department of Mathematics, University of Tennessee, Knoxville, Tennessee 37996-1300 